Recibido:

8-IV-2018

Aceptado:

26-IV-2018

Publicado en línea:

7-V-2018
Evaluación radiográfica de la presencia/agenesia de terceros molares en una población infantil Mexicana

\section{Radiographic Evaluation of the Presence/Agenesis of Third Molars in a Mexican Child Population}

Jade Viridiana San Román-Hernández DDS1; Amaury Pozos-Guillén DDS, PhD1; Ricardo Martínez-Rider DDS1; Socorro Ruiz-Rodríguez DDS, MS; Arturo Garrocho-Rangel DDS, PhD¹; Miguel Ángel Rosales-Berber DDS1

1. Especialidad en Estomatología Pediátrica, Facultad de Estomatología, Universidad Autónoma de San Luis Potosí; San Luis Potosí, S.L.P., México.

Autor para correspondencia: Dr. Miguel Ángel Rosales-Berber - miguel.rosales@uaslp.mx

RESUMEN: En el ser humano, el tercer molar es el órgano dentario que más variaciones presenta durante su desarrollo embriológico, y es causa de diversas alteraciones y malestares durante su proceso de erupción. Se ha señalado que la agenesia del tercer molar tiene una prevalencia entre 9 y 37\%. El objetivo del presente estudio transversal comparativo fue evaluar radiográficamente la presencia/ausencia de gérmenes de terceros molares (G3M) en pacientes pediátricos de origen mexicano. Se analizaron 513 radiografías panorámicas de pacientes que asistieron al Posgrado en Estomatología Pediátrica de la Universidad Autónoma de San Luis Potosí (México), durante los años 2011 a 2017. Se tomaron como criterios de inclusión, pacientes entre los 7 y 18 años de edad, sexo indistinto; se excluyeron pacientes con antecedentes de extracción de alguno de los terceros molares, tratamiento ortodóntico previo, enfermedades congénitas y/o síndromes asociados. Se compararon las frecuencias y prevalencias de agenesia de terceros molares, en total y por cuadrantes, comparando por sexo y grupo de edad. 245 radiografías panorámicas correspondieron al sexo femenino y 268 al masculino. La prevalencia total de agenesia del G3M fue estimada en casi $56 \%$, y el grupo de edad de 7-8 años mostró el mayor porcentaje; sin embargo, cuando este grupo de edad se excluyó del análisis, dicha prevalencia disminuyó a 27.3\%. Los cuadrantes mandibulares mostraron mayor porcentaje de agenesia. No hubo diferencia estadística significativa en la comparación por sexo. La prevalencia total de agenesia del G3M reportada en el presente estudio se encuentra muy por encima de los parámetros internacionales. Es muy posible que la falta de visualización radiográfica de agenesia de los terceros molares haya sido errónea en algunos casos 
debido a la ausencia frecuente de indicios de calcificación de este diente durante las edades tempranas (7-8 años). El análisis ajustado proporcionó un valor de prevalencia más acorde con los estándares reconocidos por la literatura dental.

\section{PALABRAS CLAVE: Agenesia; Prevalencia; Tercer molar.}

ABSTRACT: In the human being, third molar is the tooth that exhibits more variants during its embryologic development, usually causing diverse anomalies and discomfort when erupting to the oral cavity. It has been pointed out that the prevalence of third molar agenesis is between 9 to $37 \%$. The aim of the present comparative crosssectional study was to radiographically assess the presence/absence of third molar germs (G3M) in a sample of pediatric patients of Mexican origin. A total of 513 panoramic radiographs were analyzed from patients attending the Pediatric Dentistry Postgraduate Program ( San Luis Potosí University, México), during the years 2011 to 2017. Inclusion criteria were patients between 7 and 18 years old, any gender; children with antecedents of a third molar extraction, previous orthodontic treatment, or with an associated congenital or systemic condition were excluded. Total prevalence G3M agenesis was calculated. Then, statistical comparisons of agenesia proportions per dental quadrant, gender, and age group were performed. 245 panoramic radiographs corresponded to the female gender, while 268 belonged to the male gender. The total prevalence of G3M agenesia was estimated in nearly $56 \%$, and the $7-8$ years old group exhibited the highest proportion; however, when this age group was excluded from the analysis, such prevalence decreased to $27.3 \%$. Both mandibular quadrants showed higher proportions of G3M agenesia. There were no significant differences between genders. The total prevalence reported in the present study was found to be well above regarding the international parameters. It is quite possible that lack of radiographic visualization of G3M had been inaccurate in some cases due to the common absence of calcification traces from those teeth during early ages (7-8 years old). The adjusted analysis provided a prevalence value more in line with the recognized standards in the dental literature.

KEYWORDS: Agenesia; Prevalence; Third molar.

\section{INTRODUCCIÓN}

Durante la odontogénesis pueden ocurrir anomalías de diferentes tipos que afectan la forma, tamaño, estructura y número de los dientes en formación. Entre estas anomalías se encuentran las agenesias dentales 0 ausencia de uno 0 más gérmenes dentarios $(1,2)$. La agenesia dental se considera como la anomalía del desarrollo oral y craneofacial más común del ser humano, cuyo origen es multifactorial, e influenciada por factores genéticos, ambientales, patológicos y evolutivos (3). La agenesia dental puede comprometer a varios órganos dentarios de la dentición humana; sin embargo, es indudable que el tercer molar es el que exhibe la mayor prevalencia de agenesia a nivel mundial (entre 9 al 37\%) (3-5); en México, tal prevalencia se ha reportado alrededor del $25 \%(6,7)$.

El tercer molar, también llamado "molar del juicio" o "molar cordal", se localiza en la parte más distal o posterior de los maxilares (8). Su proceso 
de formación comienza aproximadamente a los 3 años. A los 6 años hace aparición la papila y la pared folicular del germen del tercer molar (G3M) (9) y la edad promedio de inicio de calcificación del G3M comienza a los 8-9 años, completando la formación radicular entre 16 y 25 años (10). Algunos autores han asociado a la agenesia dental del tercer molar con los diferentes genes que codifican los factores de crecimiento, y que están encargados de regular el funcionamiento celular y también de determinar la forma, tamaño y el número de dientes. Esta anomalía se asocia a una alteración del proceso de formación de la lámina dentaria, lo que ocurre entre el quinto mes de vida intrauterina y el nacimiento $(11,12)$. Por otra parte, enfermedades como raquitismo, sífilis congénita, deficiencias nutricionales, tuberculosis, escarlatina, rubeola, radiaciones y traumas pueden ser igualmente responsables de la agenesia dental (1).

De acuerdo a varias teorías, la agenesia dental de este órgano dentario se ha atribuido también a factores evolutivos. Por ejemplo, la teoría filogenética, menciona que el desarrollo y la evolución de la especie humana a lo largo de miles de años ha producido diversos cambios en la dentición (13); o la teoría de la reducción terminal dentaria de Adloff, que teoriza la futura desaparición del tercer molar en la especie humana, como consecuencia de la disminución de la actividad masticatoria y la modificación gradual de los hábitos alimenticios (4); se ha comprobado que estos factores han contribuido a la disminución progresiva de la longitud del espacio retromolar, particularmente en la mandíbula (14). Sin embargo, otros autores descartan estas teorías y consideran a la agenesia del tercer molar como una anomalía del desarrollo causado por un proceso de mutación y selección basado en la herencia (4).

Generalmente, el proceso de erupción del tercer molar es intrincado y se asocia con algunas anomalías como son retención intraósea, dolor, inflamación, procesos infecciosos, reabsorción de la raíz del segundo molar permanente, malposición dental, enfermedad periodontal localizada, formación de quistes, dolor de la articulación temporomandibular, además de recidivas de tratamientos ortodónticos $(5,6,15)$.

En este contexto, es necesario que el Estomatólogo Pediatra y el Dentista General cuenten con el conocimiento suficiente acerca de las ventajas de la detección oportuna del G3M, además de las anomalías asociadas, para planeación de un manejo terapéutico efectivo. Por lo tanto, el objetivo del presente estudio fue evaluar radiográficamente la presencia/ausencia de terceros molares en pacientes pediátricos de una población mexicana. Además se realizaron comparaciones entre prevalencias de agenesia del G3M por sexo y por grupos de edad.

\section{MATERIALES Y MÉTODOS}

El diseño del estudio fue de tipo transversal analítico. Se analizaron 513 radiografías panorámicas tomadas de pacientes pediátricos a partir de los 7 años, quienes asistieron a la clínica del Posgrado en Estomatología Pediátrica de la Universidad Autónoma de San Luis Potosí, México, durante el periodo comprendido entre el 1 de enero 2011 al 31 de julio 2017. Se determinó a los 7 años como la edad inicial para la evidencia radiográfica del inicio del proceso de calcificación del tercer molar, reportada entre los 5 a 9 años en la literatura dental $(4,10,16)$. La edad máxima de los pacientes fue de 18 años.

Los criterios de inclusión fueron los siguientes: Radiografías panorámicas obtenidas de pacientes de 7 a 18 años de edad, de cualquier sexo, con historia clínica completa. Se excluyeron pacientes con antecedentes de extracción de terceros molares, con tratamiento ortodóntico previo, presencia de enfermedades congénitas y/0 síndromes asociados. De cada paciente se registraron la información demográfica, antecedentes heredo- 
familiares y antecedentes de enfermedad sistémica. Se calcularon las frecuencias y porcentajes (prevalencia) de agenesia del tercer molar por cada cuadrante en la radiografía panorámica. También se realizaron comparaciones por sexo y por grupos de edad. Así, se clasificó a los pacientes en 4 grupos de acuerdo a su edad: Grupo A (7-8 años), Grupo B (9-10 años), Grupo C (11-12 años), Grupo D (13-14 años) y Grupo E (>15).

Los datos fueron tabulados y almacenados en una base de datos (Excel). La evaluación de cada radiografía panorámica se realizó de manera sistemática, revisando solamente 20 radiografías por día, con el fin de que la observación no se viera sesgada por el cansancio visual del observador.

Para el análisis estadístico comparativo entre los grupos (cuadrantes, sexo y grupos de edad) se utilizó la prueba de Chi-cuadrada, con un nivel de significancia de 0.05 .

\section{RESULTADOS}

Considerando la muestra total estudiada se observó que 227 pacientes presentaron los 4 G3M, mientras que 266 presentaron agenesia de por lo menos un G3M, para una prevalencia de agenesia de $55.75 \%$. Sin embargo, al excluir del análisis al grupo de edad de 7-8 años dicha prevalencia disminuye a 27.3\%. La Tabla 1 muestra la distribución por sexo de la muestra de pacientes incluidos en el estudio.

La presencia/agenesia de tercer molar y su distribución por cuadrante se exhibe en la Tabla 2. Los cuadrantes superiores mostraron una mayor prevalencia de agenesia dental, particularmente en el lado izquierdo.

En relación a la frecuencia de agenesias del G3M por paciente, 161 pacientes exhibieron ausencia de los 4 gérmenes, lo que representó el $31.39 \%$ del total de la muestra estudiada (Tabla 3).

En el análisis comparativo por sexo se obtuvo un valor de $\mathrm{p}=0.63$ (Chi-cuadrada); por lo tanto, no existió diferencia significativa entre las niñas y niños en cuanto a la agenesia del G3M (Tabla 4).

Para propósitos comparativos entre los 5 grupos de edad estudiados, se empleó también la prueba estadística Chi-cuadrada, con la que se obtuvo un valor de $p<0.001$, lo que indica que sí existe una diferencia significativa entre los grupos de edad. Se puede observar que casi la mitad del grupo de edad más joven (7-8 años) mostró ausencia de los 4 gérmenes (Tabla 5). 
Tabla 1. Distribución de los pacientes por sexo.

\begin{tabular}{ccc}
\hline Sexo & Frecuencia & $\%$ \\
\hline Femenino & 245 & 47.76 \\
Masculino & 268 & 52.24 \\
Total & 513 & 100 \\
\hline
\end{tabular}

Tabla 2. Presencia/agenesia por cuadrantes.

\begin{tabular}{ccc}
\hline Cuadrante Superior Derecho & & \\
\hline Agenesia del germen & 253 & $49.33 \%$ \\
Presencia del germen & 260 & $50.77 \%$ \\
Total & 513 & $100 \%$ \\
\hline Cuadrante Superior Izquierdo & & \\
\hline Agenesia del germen & 260 & $50.68 \%$ \\
Presencia del germen & 253 & $49.32 \%$ \\
Total & 513 & $100 \%$ \\
\hline Cuadrante Inferior Derecho & & $35.28 \%$ \\
\hline Agenesia del germen & 181 & $64.72 \%$ \\
Presencia del germen & 332 & $100 \%$ \\
Total & 513 & \\
\hline Cuadrante Inferior Izquierdo & & $35.28 \%$ \\
\hline Agenesia del germen & 181 & $64.72 \%$ \\
Presencia del germen & 332 & $100.00 \%$ \\
Total & 513 & \\
\hline
\end{tabular}

Tabla 3. Distribución de frecuencias de agenesias dentales.

\begin{tabular}{cc}
\hline Número de agenesias & Frecuencia (\%) de pacientes \\
\hline 0 & $227(44.25)$ \\
1 & $21(4.09)$ \\
2 & $80(15.59)$ \\
3 & $24(4.68)$ \\
4 & $161(31.39)$ \\
Total & $513(100)$ \\
\hline
\end{tabular}


Tabla 4. Distribución de las ausencias de tercer molar por sexo.

\begin{tabular}{|c|c|c|c|c|c|c|}
\hline Sexo & $\begin{array}{c}0 \\
(\%)\end{array}$ & $\begin{array}{c}1 \\
(\%)\end{array}$ & $\begin{array}{c}2 \\
(\%)\end{array}$ & $\begin{array}{c}3 \\
(\%)\end{array}$ & $\begin{array}{c}4 \\
(\%)\end{array}$ & $\begin{array}{c}\text { TOTAL } \\
\text { (\%) }\end{array}$ \\
\hline Femenino & $\begin{array}{c}115 \\
(46.94)\end{array}$ & $\begin{array}{c}9 \\
(3.67)\end{array}$ & $\begin{array}{c}33 \\
(13.47)\end{array}$ & $\begin{array}{c}10 \\
(4.08)\end{array}$ & $\begin{array}{c}78 \\
(31.84)\end{array}$ & $\begin{array}{c}245 \\
(47.76)\end{array}$ \\
\hline \multirow[t]{2}{*}{ Masculino } & $\begin{array}{c}112 \\
(41.79)\end{array}$ & $\begin{array}{c}12 \\
(4.48)\end{array}$ & $\begin{array}{c}47 \\
(17.54)\end{array}$ & $\begin{array}{c}14 \\
(5.22)\end{array}$ & $\begin{array}{c}83 \\
(30.97)\end{array}$ & $\begin{array}{c}268 \\
(52.24)\end{array}$ \\
\hline & $\begin{array}{c}227 \\
(44.25)\end{array}$ & $\begin{array}{c}21 \\
(4.09)\end{array}$ & $\begin{array}{c}80 \\
(15.59)\end{array}$ & $\begin{array}{c}24 \\
(4.68)\end{array}$ & $\begin{array}{c}161 \\
(31.39)\end{array}$ & $\begin{array}{c}513 \\
(100)\end{array}$ \\
\hline
\end{tabular}

Tabla 5. Distribución por número de agenesias del tercer molar y por grupo de edad.

\begin{tabular}{|c|c|c|c|c|c|c|}
\hline $\begin{array}{c}\text { Grupos de } \\
\text { edad }\end{array}$ & $\begin{array}{c}0 \\
(\%)\end{array}$ & $\begin{array}{c}1 \\
(\%)\end{array}$ & $\begin{array}{c}2 \\
(\%)\end{array}$ & $\begin{array}{c}3 \\
(\%)\end{array}$ & $\begin{array}{c}4 \\
(\%)\end{array}$ & $\begin{array}{c}\text { Total de } \\
\text { pacientes }\end{array}$ \\
\hline $\begin{array}{c}\text { A } \\
(7-8)\end{array}$ & $\begin{array}{c}41 \\
(21.93)\end{array}$ & $\begin{array}{c}8 \\
(4.28)\end{array}$ & $\begin{array}{c}35 \\
(18.72)\end{array}$ & $\begin{array}{c}10 \\
(5.35)\end{array}$ & $\begin{array}{c}93 \\
(49.73)\end{array}$ & 187 \\
\hline $\begin{array}{c}\text { B } \\
(9-10)\end{array}$ & $\begin{array}{c}112 \\
(50.00)\end{array}$ & $\begin{array}{c}10 \\
(4.46)\end{array}$ & $\begin{array}{c}36 \\
(16.07)\end{array}$ & $\begin{array}{c}10 \\
(4.46)\end{array}$ & $\begin{array}{c}56 \\
(25.00)\end{array}$ & 224 \\
\hline $\begin{array}{c}\mathrm{C} \\
(11-12)\end{array}$ & $\begin{array}{c}52 \\
(68.42)\end{array}$ & $\begin{array}{c}3 \\
(3.95)\end{array}$ & $\begin{array}{c}8 \\
(10.53)\end{array}$ & $\begin{array}{c}2 \\
(2.63)\end{array}$ & $\begin{array}{c}11 \\
(14.47)\end{array}$ & 76 \\
\hline $\begin{array}{c}D \\
(13-14)\end{array}$ & $\begin{array}{c}11 \\
(91.67)\end{array}$ & $\begin{array}{c}0 \\
(0.00)\end{array}$ & $\begin{array}{c}0 \\
(0.00)\end{array}$ & $\begin{array}{c}1 \\
(8.33)\end{array}$ & $\begin{array}{c}0 \\
(0.00)\end{array}$ & 12 \\
\hline $\begin{array}{c}E \\
(>15)\end{array}$ & $\begin{array}{c}11 \\
(78.57)\end{array}$ & $\begin{array}{c}0 \\
(0.00)\end{array}$ & $\begin{array}{c}1 \\
(7.14)\end{array}$ & $\begin{array}{c}1 \\
(7.14)\end{array}$ & $\begin{array}{c}1 \\
(7.14)\end{array}$ & 14 \\
\hline TOTAL & 227 & 21 & 80 & 24 & 161 & 513 \\
\hline
\end{tabular}




\section{DISCUSIÓN}

El presente estudio mostró que la agenesia de uno o más terceros molares, con una prevalencia de $55.75 \%$, es más frecuente que la presencia de los mismos (44.25\%). Este valor de prevalencia está muy por encima de los reportados en otros trabajos mencionados en la literatura. Aunque sus resultados son variables debido a las diferentes características raciales de cada muestra y a la metodología empleada, la mayoría de ellos coincide en que la prevalencia de agenesia del G3M se encuentra alrededor del 25 al 30\%. Por ejemplo, Killinc et al., (17) realizaron un estudio radiográfico retrospectivo en una muestra de 773 radiografías de pacientes de 12-18 años de edad, residentes en Turquía, en el que se concluyó que el $23.3 \%$ de la muestra presentaban agenesia de 1 a 4 gérmenes de terceros molares. Hegde et al., (18) estudió en la India una muestra de 700 niños, concluyendo que el $34.1 \%$ presentaban agenesia de terceros molares. En otro estudio realizado por Herrera-Atoche et al., en Yucatán (México) se evaluaron 670 radiografías de pacientes de 9 a 20 años que acudían al departamento de Ortodoncia se reportó una prevalencia de agenesia del tercer molar de casi $26 \%$ (7).

En el presente estudio, la alta prevalencia encontrada puede ser explicada por la errónea detección de agenesia de G3M, es decir como un dato falso negativo, en algunos casos. Es frecuente que durante las edades tempranas sea imposible visualizar radiográficamente la presencia de los G3M, debido a la ausencia de indicios de calcificación en estos dientes. Sin embargo, al eliminar los valores del grupo de edad de 7-8 años, se produjo un valor de prevalencia de $27.3 \%$, más acorde con los estándares reconocidos por la literatura dental. Al respecto, se acepta en general que la edad promedio de detección del G3M oscila entre los 7 a 9 años de edad, aunque algunos autores han reportado casos ocasionales en los que se ha logrado visualizar radiográficamente al G3M ya desde los 5 años (10). Es por eso que, para poder determinar con mayor exactitud la presencia o agenesia de los terceros molares, el paciente pediátrico debe ser monitorizado radiográficamente hasta los 14 años de edad $(4,16)$. En cuanto a la agenesia del G3M por arcada dentaria, el presente estudio determinó que el maxilar superior mostró mayor prevalencia de esta anomalía. Resultados similares han sido reportados en la literatura. En un estudio se encontró una diferencia $(p<0.001)$ en el porcentaje de agenesia de terceros molares en el maxilar (14.3\%) comparado con la mandíbula (9.6\%) (17). En otro estudio similar realizado por Ibarra (19), se informó que un grupo de 1046 pacientes de 13 a 14 años, fue más común observar agenesias en el arco maxilar que en la mandíbula.

En relación a la prevalencia por sexo, el presente estudio no encontró diferencias significativas entre los grupos. Al respecto, un trabajo publicado que incluyó 670 pacientes $(34.64 \%$ masculinos $n=239$ y $65.36 \%$ femeninos $n=451$ ), de 9 a 20 años de edad tampoco encontró diferencia estadísticamente significativa entre ambos sexos $(p=0.139)(7)$.

De acuerdo a grupos de edad, los resultados del presente trabajo indican que existió una diferencia significativa entre ellos $(p<0.001)$. El grupo que presentó una mayor frecuencia agenesias de terceros molares fue el grupo de 7-8 años; sin embargo, es necesario considerar que los grupos de 13-14 años y 15-18 años presentaron una menor cantidad de radiografías evaluadas. Resultados similares fueron encontrados por Bartolomè-Villar et al., (20), quienes realizaron un estudio con una muestra de niños de 6-17 años.

En relación a la comparación por cuadrantes realizada aquí, el cuadrante superior izquierdo mostró mayor prevalencia de agenesia del tercer molar. Coincidentemente, en una investigación realizada por Lucas et al., en la que se observaron 
124 radiografías panorámicas, se concluyó que el cuadrante con más agenesias fue también el superior izquierdo. Además, se observó un mayor desarrollo en los G3M en el cuadrante inferior izquierdo (10).

Las consecuencias que provocan la variabilidad anatómica y dirección de erupción del tercer molar vuelven a este tópico clínico un tema de gran preocupación e interés para el Odontólogo Pedíatra La detección radiográfica de la presencia del germen del tercer molar en sus fases iniciales de calcificación previa a su erupción es fundamental, por lo que se recomienda obtener una radiografía panorámica a partir de los 7 u 8 años de edad con la finalidad de vigilar su dirección de erupción, así como para prevenir o tratar oportunamente aquellas complicaciones asociadas.

En conclusión, La prevalencia total de agenesia del G3M reportada en el presente estudio se encuentra muy por encima de los parámetros internacionales. Es muy posible que la falta de visualización radiográfica de agenesia de los terceros molares haya sido errónea en algunos casos debido a la ausencia frecuente de indicios de calcificación de este diente durante las edades tempranas (7-8 años). El análisis ajustado proporcionó un valor de prevalencia más acorde con los estándares reconocidos por la literatura dental.

\section{REFERENCIAS}

1. Chappuzeau E., Cortés D. Anomalías de la dentición en desarrollo: Agenesias y supernumerarios. Rev Dent Chile. 2008; 99 (2): 3-8.

2. Marchena-Rodríguez L., Fernández-Ortega C. Etiología de las agenesias dentales. REDOE; 2015 [consultado 30 de enero de 2017]; Disponible en: http://www.redoe. com/ver.php?id=192

3. Botina C. J., Rodríguez L. F., Cepeda E. C., Zabala D. F., González-Colmenares G. Frecuencia de agenesias de terceros molares: Relación con el tamaño mandibular. Rev Nac Odontol. 2012; 8 (15): 52-6.

4. García-Hernández F.., Toro-Yagui O., VegaVidal M., Verdejo-Meneses M. Agenesia del tercer molar en jóvenes entre 14 y 20 años de edad, Antofagasta, Chile. Int J Morphol. 2008; 26 (4): 825-32.

5. Khan-Sujon M., Khursheed-Alam M., Abdul-Rahman S. Prevalence of third molar agenesis: Associated dental anomalies in non-syndromic 5923 patients. PLoS ONE. 2016; 11 (8): e0162070.

6. Silva-Meza R. Radiographic assessment of congenitally missing teeth in orthodontic patients. Int J Paediatr Dent. 2003; 13 (2): 112-6. 
7. Herrera-Atoche J. R., Colomé-Ruiz G. E., Escoffié-Ramírez M. Agenesia de terceros molares, prevalencia, distribución $\mathrm{y}$ asociación con otras anomalías dentales. Int J Morphol. 2013; 31 (4): 1371-5.

8. Colorado-Pinillo M., Huitzil-Muñoz E. Agenesia de terceros molares en pacientes del Centro del estado de Veracruz. Rev TAME. 2015; 4 (11): 379-82.

9. Armand Lorié M., Legrá Silot E., Ramos de la Cruz M., Matos Armand F. Terceros molares retenidos. Rev Inf Cient. 2015; 92 (1): 995-1010.

10. Lucas G. V., Martínez S. E., Discacciati De Lértora S., Galiana A. V., Elizondo M. L. Detección radiográfica de anomalías y patologías dentarias y determinación del grado de desarrollo de los terceros molares en niños en entre 5 y 10 años. Comunicaciones Científicas y Tecnológicas 2008. Disponible en; http://www.unne.edu.ar/unnevieja/ investigacion/com2008/M-006.pdf

11. Echeverri-Escobar J., Restrepo-Perdomo L. A., Vásquez-Palacio G., Pineda-Trujillo N., Isaza-Guzmán D. M., Manco-Guzmán H. A., Marín-Botero M. L. Agenesia dental: Epidemiología, clínica y genética en pacientes antioqueños. Av Odontoestomatol. 2013; 29 (3): 119-30.

12. Kolenc-FuséF.J.Agenesias dentarias: en busca de las alteraciones genéticas responsables de la falta de desarrollo. Med Oral Patol Oral Cir Bucal. 2004; 9 (5): 385-95.

13. Calvo-Hernández I., Mourelle-Martínez M. R. Estudio clínico de las agenesias dentarias en una población infantil. Universidad Compútense de Madrid. Disponible en: http://eprints.
ucm.es/19912/1/Isabel_Calvo_HernándezEstudio_cl\%C3\%ADnico_de_las_agenesias dentarias_en_una_población.pdf

14. Pamplona J. La mandíbula y el tercer molar (Tesis Doctoral). Universidad de Granada. Granada, España. 44, 1983.

15. Mursulí M., Rodríguez H., Landa L., Hernández M. Anomalías dentales. Gac Med Espirit. 2006; 8 (1): 366-88.

16. Prieto J. L. Third molar maturation and age assessment. Evolution and state of the art. Cuad Med Forense, 2008; 14 (51): 11-24.

17. Killinc G., Akkemik OK., Candan U., Evcil MS., Ellidokuz H. Agenesis of third molars among Turkish children between the ages of 12 and 18 years: A retrospective radiographic study. J Clin Pediatr Dent. 2017; 41 (3): 243-7.

18. Hegde S., Patosia A., Dixit U. Staging of third molar development in relation to chronological age of 5-16 year old Indian children. Forensic Sci Int. 2016; 269 (3): 63-9.

19. Ibarra A. Tesis Prevalencia de agenesia de terceros molares y estadio de Nolla en pacientes que acuden al CEOB; Centro de Estudios de Ortodoncia del Bajio. [Tesis doctoral en internet]. Citado el 30 de septiembre del 2013. 48 p. Disponible en: https://es.slideshare.net/alan_master/dr-alanibarra-prevalencia-de-agenesia-de-tercerosmolares-y-estadio-de-nolla-en-pacientesque-acuden-al-ceob

20. Bartolomè-Villar B., Barberia-Leache E., De Nova-García J., Beltri-Orta P. Desarrollo y agenesia del tercer molar en una población de niños y adolescentes españoles. Estudio de la agenesia (II). Odontol Pediatr. 1994; 3 (3): 107-12. 